\title{
Genotypic diversity of Legionella pneumophila in environmental and clinical strains assessed by Sequence-Based Typing, in association with retrospective clinical surveillance in Northern Italy
}

\author{
Annalisa Bianchi', Fabrizio Ernesto Pregliasco², Michela Consonni' ${ }^{1}$, Marina Tesauro' \\ ${ }^{1}$ Dipartimento di Scienze Biomediche Chirurgiche Odontoiatriche Università degli Studi di Milano, Italy \\ 2 Dipartimento di Scienze Biomediche per la Salute Università degli Studi di Milano, Italy
}

\begin{abstract}
Bianchi A, Pregliasco FE, Consonni M, Tesauro M. Genotypic diversity of Legionella pneumophila in environmental and clinical strains assessed by Sequence-Based Typing, in association with retrospective clinical surveillance in Northern Italy. Ann Agric Environ Med. 2016; 23(2): 248-253. doi: $10.5604 / 12321966.1203885$
\end{abstract}

\begin{abstract}
Objective. The study aimed to evidence previous cases of legionellosis or proven or suspected pneumonia in seven hospital facilities for the mentally disabled in Northern Italy, where no clinical surveillance had been previously carried out. An additional aim was to highlight the occurrence of strains of Legionella pneumophila of clinical and environmental origin by Sequence-Based Typing (SBT), comparing them to world surveillance.

Materials and method. A clinical survey was perfomed from 2003-2012, analyzing 615 medical records for hospital- and community-acquired pneumonia, with particular attention to legionellosis. Clinical $(n=4)$ and environmental $(n=25)$ isolates of Legionella pneumophila, isolated in the same period (2003-2012), were characterized by SBT and the Sequence Types (STs) compared with the European Working Group for Legionella Infections (EWGLI) database.

Results. Surveillance revealed that there were seven detected cases of legionellosis; most pneumonia cases could not be confirmed by diagnostic tests because of the disabilities of the patients and their lack of cooperation. The same ST was found in two of the clinical strains and also in a corresponding environment, i.e. ST685 and ST16, and two clinical strains belonging to the same ST (ST1). The other environmental strains were isolated in department with confirmed/suspected clinical cases. Five other STs found in this study were new to the database: ST685 was isolated both from a patient and from water; ST694, ST1181, ST1370 and ST1371 have not been described previously.

Conclusions. The study confirmed that the routine collection and analysis of environmental strains may be an important strategy for preventing sporadic and epidemic cases of legionellosis, in association with clinical surveillance.
\end{abstract}

Key words

Legionella pneumophila, genotyping, clinical surveillance, mental disabilities, long-term care facilities

\section{INTRODUCTION}

Legionnaires' disease is a type of pneumonia caused by Legionella spp., which are environmental Gram-negative bacteria. Pontiac fever is a febrile, non-pneumonic, shortincubation period, non-fatal disease associated with exposure to water-borne aerosols containing Legionella spp. bacteria. The great majority of cases of Legionnaires' disease (LD) are caused by Legionella pneumophila, in particular $L$. pneumophila serogroup 1. L. pneumophila was recognized about 36 years ago $[1,2]$. Clinical signs are not specific, which makes prompt and accurate laboratory diagnosis crucial [3].

In recent years, numerous phenotypic and genotypic typing methodologies have been developed and applied to the epidemiological typing of L. pneumophila [4]. These include monoclonal antibody (MAb) subgrouping as a rapid screening method, and various genotyping methods, such as restriction fragment length polymorphism (RFLP)

Address for correspondence: Marina Tesauro, Dipartimento di Scienze Biomediche Chirurgiche Odontoiatriche Università degli Studi di Milano, Via C.Pascal 36, 20133 Milan, Italy

E-mail: marina.tesauro@unimi.it

Received: 29 January 2015; accepted: 23 October 2015 analysis, amplified fragment length polymorphism (AFLP), and pulsed-field gel electrophoresis (PFGE). In contrast to band-based typing methods that are difficult to standardize, the sequence-based typing (SBT) system has the potential to achieve excellent typeability, interlaboratory reproducibility and epidemiologic concordance $[3,5,6,7]$.

Several studies have attempted to discriminate between more pathogenic and less pathogenic strains by demonstrating the presence of genes associated with virulence genes. More than 70 serogroups of Legionella and 16 of L. pneumophila and also many sequence types (about 1,300 of L. pneumophila) are known, but little is known about differences in virulence or the ability of strains to invade the host cell $[8,9]$. Other studies have assessed the possible threshold levels of environmental contamination, considering the risk of infection of different types of patients, but these require further investigation.

In Italy, a guideline was issued in 2000 by the Ministry of Health [10], only recently revised in a new document with an indication of the thresholds for intervention in hospitals and nursing homes [11]. There are also local documents for prevention and control in hospitals with severely immunocompromised patients, with the integration of the mentally ill as a category of patients at risk $[12,13]$. 
For these reasons, the presented study investigated environmental exposure to L. pneumophila in individuals with permanent neurologic and mental disabilities of varying degrees:

- residents who were completely non-self-sufficient, those with serious physical disability and mental retardation which implied a requirement for nursing care for their primary needs;

- residents who were partially self-sufficient, those able to carry out activities outside their ward, but presenting a degree of mental impairment which resulted in difficulties with taking care of themselves.

\section{OBJECTIVE}

The aim of the study was to use a new approach that takes into account the needs of patients who are particularly at risk of contracting this disease, those with the greatest difficulties in submitting to routine diagnosis and maintaining the delicate psychological balance necessary to avoid compromising or causing a general deterioration in their condition. For this reason, a pilot study of clinical surveillance (with retrospective analysis of medical records and raise awareness in physicians on these issues) was prepared, supported by environmental monitoring, in addition to routine water sampling in the structures. The scope was to evaluate evidence for previous cases of legionellosis or with proven or suspected pneumonia, and to study the occurrence of strains of Legionella pneumophila of clinical and environmental origin by Sequence-Based Typing (SBT), comparing them to world surveillance.

\section{MATERIALS AND METHOD}

Clinical and environmental surveillance. Six nursing homes for psychiatric patients (one with about 1,000 beds and the other five branches having approximately 100 beds each) and a hospital that accommodates patients requiring specific long-term interventions or care in specialized departments (medicine, surgery, cardiology, pulmonology, nephrology) were considered in this study.

The patients assisted in these facilities suffer from mental retardation of varying degrees (mild to very serious), and may be affected by rare diseases such as West, Lennox, X-Fragile, Cri-Du-Chat, MICRO, Down, Williams, Graham-Little, Wolf-Hirshhorn, and Charge syndrome.

The considered population suffers from neurologic or psychiatric disorders, such as motor disabilities (primarily cerebral palsy), aberrant behaviour, epilepsy, and mood and impulse control disorders often associated with general medical problems.

In these structures a pilot study was developed that involved a system of clinical surveillance and environmental monitoring. Briefly, a clinical retrospective survey was undertaken (2003-10), which aimed to analyze hospital and community acquired pneumonia, according to the CDC definition [14], with particular attention to LD. Altogether, 615 medical records of institutionalized patients in health facilities were examined to identify cases of legionellosis, detected or suspected.
The clinical data of the patients was collected, etiological diagnosis and details of the diagnostic course (radiological, serological and cultural examinations).

The seven selected health facilities were subjected to routine investigation of control of legionellosis since 2002 in hot and cold water, which was sampled according to Italian guidelines [10]. Isolation of Legionella spp. was performed by the cultural method according to the ISO11731-2 standard technique, as previously described [ISO, 15]. In this way, 98 environmental isolates of L. pneumophila were isolated and stored in a strains collection, 25 of them selected for the SBT.

Environmental strains originating from wards with a higher number of nosocomial pneumonia with an unidentified etiology, and from wards with patients with serious risk factors that increase the probability of LD or pneumonia (i.e. patients with serious mental retardation, immunocompromised, dysphagia, enticed or with severe postural problems), were chosen, for a total of 25 strains in 12 wards of the nursing home and in 3 other wards (controls) (Tab. 1).

All four clinical strains coming from cases of confirmed or suspected legionellosis were included; clinical isolates were obtained from the strain collection of the hospital $(\mathrm{H})$. Therefore, a total of 29 strains were genotyped by SBT.

The selected strains were examined by SBT (EWGLI protocol Version 4.1) [5, 17]. Briefly, the protocol consisted of: revitalization of the strains by passage on the culture media, microbiological and serological tests, extraction of DNA, primary PCR reaction, DNA sequencing and sequence analysis.

Genomic DNA is extracted then amplified using primers targeting seven specific gene loci (i.e. flaA, pilE, asd, mip, mompS, proA, neuA). Following purification, amplicons are sequenced directly with forward and reverse primers, and the resulting consensus sequences trimmed and compared to previously assigned allele numbers using the online database. Using a pre-determined order flaA, pilE, asd, mip, mompS, proA, neuA, the combination of alleles is defined as a 7-digit allelic profile (e.g. 1,4,3,1,1,1,1) and a sequence type represented by a number (e.g. ST1).

Particularly, the results were analyzed by in-house software (Finch TV; USA Geospiza-PerkinElmer) and through the Sequence Quality Tool (SQT) available on the website (http:// www.hpa-bioinformatics.org.uk/legionella/legionella_sbt/ php/sbt_homepage.php), which allows users to directly upload standard format sequence files and analyze them, generating a quality report and automatically allelic profile and ST.

The results were compared with those in the EWGLI database [25] which collates details of L. pneumophila strains, serogroups, monoclonal antibody subgroups and SBT profiles from all over the world $[8,18,19,20,21]$, and with the authors'previous data [22, 23, 24].

\section{RESULTS}

From 2003-2012, a pilot study was performed that consisted in both clinical retrospective surveillance and environmental monitoring in six nursing homes accommodating psychiatric patients, and in one hospital, all in North Italy.

The retrospective survey aimed to analyze hospital and community pneumonia, with particular attention to LD. Environmental monitoring was carried out from two to three 
times a year according Italian guidelines (detailed data are not reported in this study [15]).

Of the 615 medical records of long-term/institutionalized patients analyzed during the retrospective surveillance (2003-10), the following were identified:

- 7 detected cases of LD (with such inclusion criteria as radiography, urinary antigen positive and bacterial isolation);

- 2 suspected LD (high antibody);

- 40 cases of detected pneumonia (with such inclusion criteria as radiography or bacterial isolation);

- 15 atypical pneumonia cases not detected by diagnostic tests;

- 205 suspected bronchopneumonia (not confirmed by diagnostic tests).

In many cases, it was not possible to make radiographs because of the difficulty to take satisfactory images of these patients and their lack of cooperation. This may have limited the diagnosis of several cases of pneumonia, therefore the majority of these cases remain unfortunately as suspected.

The strains were characterised by Sequence-Based Typing (SBT) and compared with the Sequence Types (STs) of all four clinical strains available and originating from cases of confirmed or suspected legionellosis. Also, 25 environmental strains were genotyped, selected from wards with a higher number of nosocomial pneumonia with an unidentified etiology, and from wards with patients with serious risk factors that increased the probability of LD or pneumonia (i.e. patients with serious mental retardation, immunocompromised, dysphagia, enticed or with severe postural problems) (Tab. 1).

PCR products of the expected sizes were yielded and nucleotide sequences from all seven genes fragments investigated, and obtained an allelic profile of the seven genes ( flaA, pilE, asd, mip, mompS, proA, neuA) for each isolate, comparing them to the EWGLI international database [25, 26] (Tab. 1).

Two clinical strains were correlated with the corresponding environment, i.e. Sequence Type (ST) 685 and 16, and two clinical strains in the same structure (ST1). The other environmental strains were isolated in the department with confirmed/suspected clinical cases of pneumonia or LD. The results were compared with the EWGLI database: the ST 1, $16,34,61,71,188$ and 579 were already known among clinical and nosocomial cases in the literature.

Five STs were new to the database: ST685 was isolated both from a patient and from the water; ST694, ST1181, ST 1370 and 1371 were unknown in the international database, and isolated from environmental samples. Comparing the Italian situation in the same period, ST 188 and ST 1362 were both new and came from water. Table 2 also shows the countries with isolated strains [25] having the same allelic profiles as those in the presented study in the same period.

\section{DISCUSSION AND CONCLUSIONS}

Water systems have been identified as a source of Legionella pneumonia [27]. The water plants of hospitals and health care facilities are often involved in the transmission of Legionella spp. and subsequent pneumonia in their patients characterized by compromised health conditions.
According the last report, in 2013 in Italy, there were 62 cases of nosocomial legionellosis, $4.6 \%$ of the total notified, but it is assumed that data have been greatly underestimated. Also reported were 21 cases associated with a stay in a residential facility for the elderly. [28]

The problem of under-reporting of cases was also evident in the current study that first analyzed 615 medical records of six nursing homes for the mentally disabled, and second, genotyped clinical and environmental strains by SBT and isolated in the same structures.

In the retrospective clinical surveillance performed from $2003-2012$, it was clear that there was a great underestimation of the number of cases. In most cases of pneumonia, no radiographs were made because it was difficult to take satisfactory images of these patients due to their lack of cooperation. This problem might have limited the correct diagnosis of pneumonia. Also, there were considerable difficulties in obtaining clinical strains (patients are subjected only to urinary antigen) and in etiological diagnosis (letting patients with mental retardation to undergo routine testing is often more difficult).

From a preliminary analysis of clinical surveillance data, there are more frequent cases of pneumonia in the wards with patients suffering from severe and profound mental retardation and dysphagia (cases of aspiration are frequent), compared to departments with older patients who have COPD, and children with mental retardation of varying degrees. These patients may be at risk for LD.

The utility of environmental monitoring for Legionella species remains controversial [27]. According to one strategy, routine environmental culturing of hospital water, even in the absence of known cases, is a proactive approach for the prevention of hospital-acquired Legionnaires' disease [29, $30,31]$. According to an alternative strategy proposed by the United States Centers for Disease Control and Prevention, intensive clinical surveillance without routine environmental monitoring is to be preferred [32].

Great help can also be provided from the intersection of clinical and environmental data, as in the presented study, from the genotyping of isolated strains and comparing them with international data to determine the frequency and virulence of known clones.

In particular, on patients such as those considered in this study, it would be desirable to propose - in collaboration with physicians - the application of molecular biology methods directly on respiratory samples (often carried out to help patient with symptoms, but rarely analyzed for clinical isolation, because of poor growth or selection of microorganisms) [3].

There is thus the need for further studies, also the use of other methods such as SBT, with respect to the increased occurrence in hospitals of certain strains with specific profiles, compared with others that occur in the environment, but not as frequently in clinical isolates. It is reasonable to assume that some clones are more virulent than the others, and that a select group of STs have an enhanced ability to cause legionellosis in humans [4].

The presented study correlates two clinical strains with the corresponding environmental strain (Sample Nos. 1 and 2, Nos. 5 and 6) and two clinical strains (Nos. 3 and 4).

It was also possible to observe that some ST $(1,16,34,188$, $328,421,579$ and 807 ) were found in clinical and nosocomial compartments, with a fair distribution in different countries; 
Table 1. Results for the 29 samples analyzed with Sequence Based Typing from 7 hospital facilities of Northern Italy after linical and environmental surveillance (2003-12)

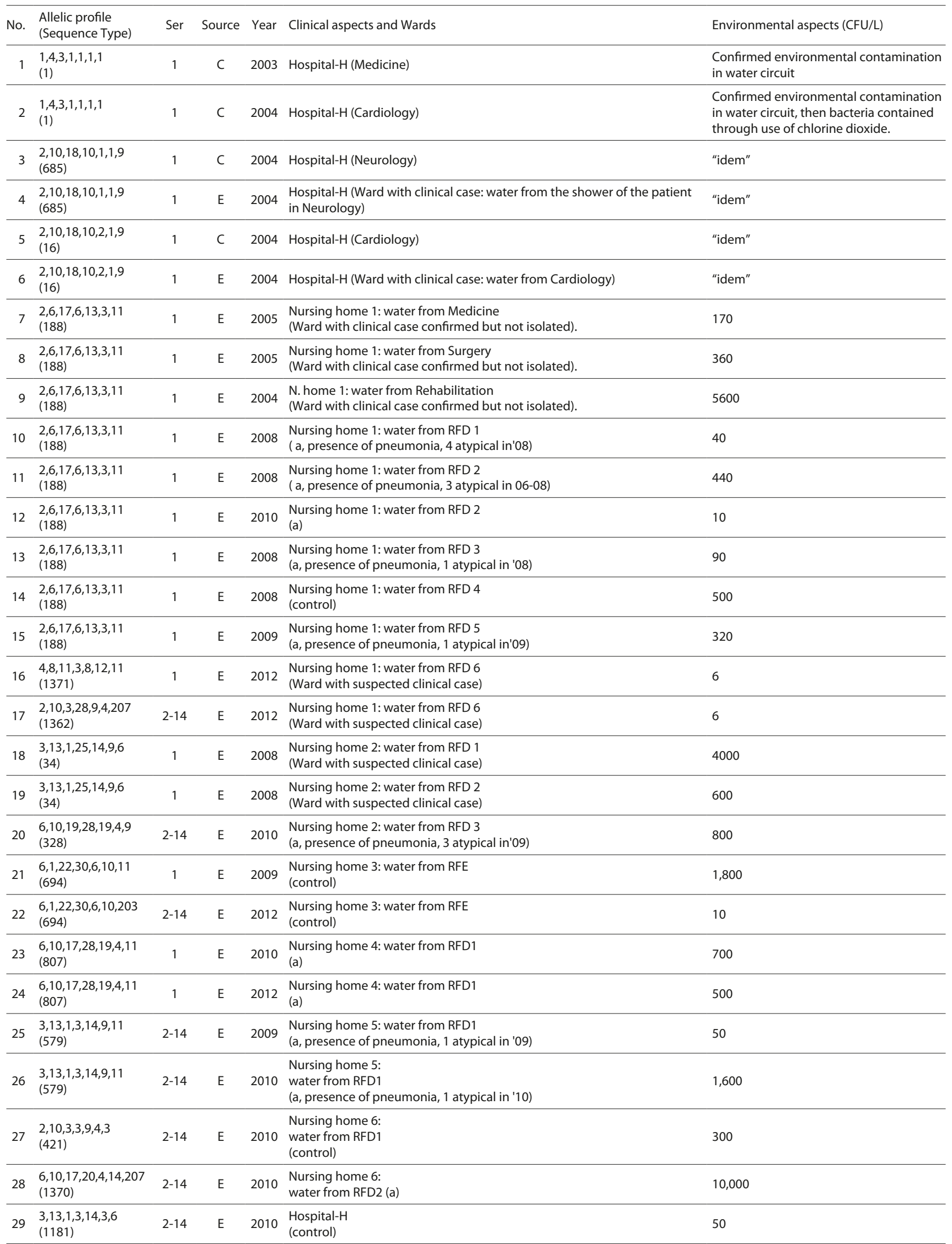

Ser-serogroup; C - clinical isolate; E - environmental isolate; RFD - Residential facility for the disabled; RFE - Residential facility for the elderly; 
Table 2. Results for the 29 samples analyzed with Sequence Based Typing and Sequence Types comparison with other regions of the world in the same period of the study

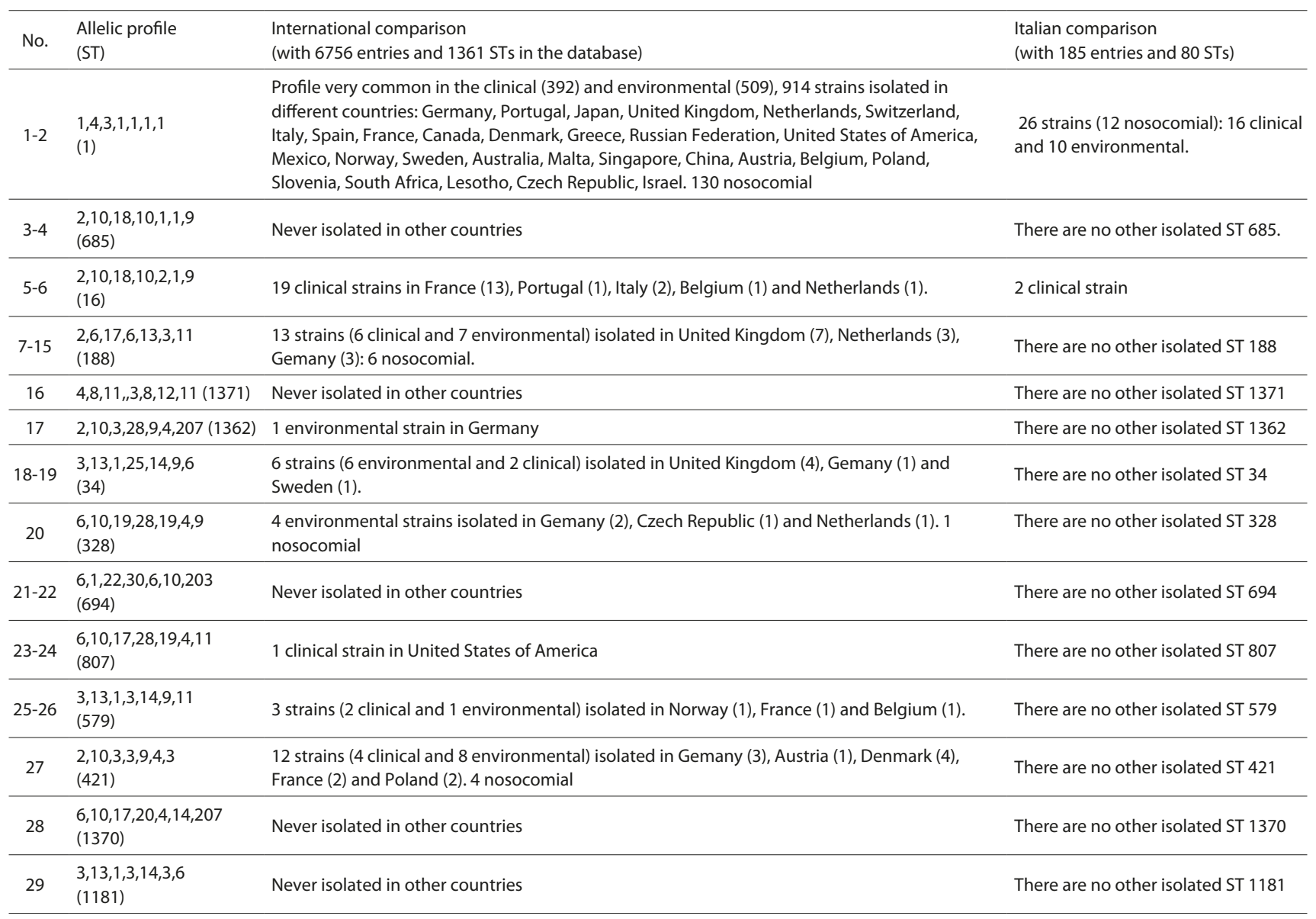

in particular, ST1 is the most commonly isolated in Europe [4]. ST 685 was not found in the EWGLI database, but its pathogenicity has been demonstrated by the concomitant isolation from a symptomatic patient and water.

In contrast, ST 694, ST 1370, ST 1371 and ST1181 were not included in the database or other source of published data in the same period of the study.

As cases of legionellosis had never been detected or suspected in the structures, it can be assumed that the profiles in question are rarer or less virulent than the other genotypes. However, given the small number of isolates studied, this hypothesis must be verified.

From comparison with time series analysis of the environmental results $[22,24]$, structure 1 was always characterized by ST 188, from which a reduction of diversity in the strains isolated from the water can be assumed. The remediation implemented since 2004 could have favoured the selection of a strain more resistant to treatment than others of the same serogroup. Despite analyzing a limited number of samples, there seems to be homogeneous colonization in each of the facilities studied, ST 579 was evidenced in nursing home No. 5 both in 2009 and 2010, or ST 694 in nursing home No.3 in 2009 and 2012, even though the presence of other Legionella (species, serogroup or ST) in low concentrations cannot be excluded. This situation differs from the hospital, where there were a greater number of strains with different allelic profiles, ST1, ST685, ST16 and ST 1181.
In agreement with European data [25], a significant portion $(20 / 24)$ of bacterial isolates belonging to clones was already known in the nosocomial department and responsible for clinical cases.

As other studies have shown $[20,21,29,30,31]$, the presented confirms that the routine analysis of environmental strains may be an important strategy for preventing outbreaks of legionellosis, in association with clinical surveillance data.

There is consensus that the issue of Legionella spp. in water is impossible to resolve; rather, we must aim to study the virulence of circulating clones and the susceptibility of patients, in addition to the containment of the bacteria and number of contaminated sites.

Containment of environmental bacteria and an advanced study on the virulence of clinical and environmental clones can be justified in the long-term, considering the serious legal implications and the image which the health facilities may have to contend with, and the known underestimation of legionellosis cases.

\section{REFERENCES}

1. Edelstein PH. Legionnaires' disease: history and clinical findings. In: Heuner K, Swanson M, Eds. Legionella - Molecular Microbiology.

Norfolk: Caister Academic Press 2008; 1-2. 
2. Gomez-Valero L, Rusniok C, Buchrieser C. Legionella pneumophila: Population genetics, phylogeny and genomics. Infect Genet Evol. 2009; 9: 727-739.

3. Ginevra C, Lopez M, Forey F, Reyrolle M, Meugnier H, Vandenesch $\mathrm{F}$, et al. Evaluation of a nested-PCR-derived sequence-based typing method applied directly to respiratory samples from patients with Legionnaires'disease. J Clin Microbiol. 2009; 47: 981-987

4. Harrison TG, Fry NK, Afshar B, Bellamy W, Doshi N, Underwood AP: Typing of Legionella pneumophila and its role in elucidating the epidemiology of Legionnaires' disease. In: Cianciotto NP, Kwaik YA, Edelstein PH et al. Eds. Legionella: state of the art 30 years after its recognition, Washington DC: ASM Press 2006: 94-99.

5. Ratzow S, Gaia V, Helbig JH, Fry NK, Luck PC. Addition of neuA, the gene encoding $\mathrm{N}$-acylneuraminate cytidylyl transferase, increases the discriminatory ability of the consensus sequence-based scheme for typing Legionella pneumophila serogroup 1 strains. J Clin Microbiol. 2007; 45: 1965-1968.

6. Farhat C, Mentasti M, Jacobs E, Fry NK, Lück C. The N-acylneuraminate cytidyltransferase gene, neuA, is heterogenous in Legionella pneumophila strains but can be used as a marker for epidemiological typing in the consensus sequence-based typing scheme. J Clin Microbiol. 2011; 49: 4052-8.

7. Mentasti M, Fry NK, Afshar B, Palepou-Foxley C, Naik FC, Harrison TG. Application of Legionella pneumophila-specific quantitative real-time PCR combined with direct amplification and sequencebased typing in the diagnosis and epidemiological investigation of Legionnaires'disease. Eur J Clin Microbiol Infect Dis. 2012; 31: 20172028.

8. Borchardt J, Helbig JH, Luck PC. Occurrence and distribution of sequence types among Legionella pneumophila strains isolated from patients in Germany: common features and differences to other regions of the world. Eur J Clin Microbiol Infect Dis. 2008; 27: 29-36.

9. Garcia-Nuñez M, Pedro-Boteta ML, Ragulla S, Sopena N, Morera J, ReyJoly C et al. Cytopathogenicity and molecular subtyping of Legionella pneumophila environmental isolates from 17 hospitals. Epidemiol Infec. 2009; 137: 188-193.

10. Italia. Ministero della Salute. Linee guida per la prevenzione e il controllo della legionellosi Gazzetta Ufficiale N. 103, 5 maggio 2000. Italian.

11. Italia, Ministero della Salute. Linee guida per la prevenzione ed il controllo della legionellosi. Approved in Conferenza Stato-Regioni on 7th May 2015- Italy

12. Regione Lombardia. Direzione Generale della Sanità. Servizio Prevenzione Sanitaria. Linee guida prevenzione e controllo della legionellosi in Lombardia. D.d.g. 24 febbraio 2009 - n. 1751. Italian.

13. Regione Piemonte. Raccomandazioni per la sorveglianza, la prevenzione e il controllo delle polmoniti da Legionella nelle strutture sanitarie piemontesi pubbliche e private. D.D. $\mathrm{n}^{\circ}$ 109; 04 marzo 2008. Italian.

14. ISO 11731-2_2004 Water quality - Detection and enumeration of Legionella part 2: Direct membrane filtration method for waters with low bacterial counts.

15. Garner JS, Jarvis WR, Emori TG Horan TC, Hughes JM. CDC definitions for nosocomial infections. Am J Infec Control. 1988; 16: 128-140.

16. Tesauro M, Bianchi A, Consonni M, Pregliasco F, Galli MG. Environmenmtal surveillance of Legionella pneumophila in two italian hospitals. Ann Ist Super Sanita. 2010; 46: 274-278.
17. Gaia V, Fry NK, Afshar B. et al. Consensus sequence-based scheme for epidemiological typing of clinical and environmental isolates of Legionella pneumophila. J Clin Microbiol. 2005; 43: 2047-2052.

18. Harrison TG, Afshar B, Doshi N, Fry NK, Lee JV. Distribuition of Legionella pneumophila serogroups monoclonal antibody subgroups and DNA sequence types in recent clinical and environmental isolates from England and Wales (2000-2008). Eur J Clin Microbiol Infect Dis. 2009; 28: 781-91.

19. Amemura-Maekawa J, Fumiaki Kura, Bin Chang and Haruo Watanabe. Legionella pneumophila Serogroup 1 isolates from cooling towers in Japan from a distinct genetic cluster. Microbiol Immunol. 2005; 49: 1027-1033.

20. Chasqueira MJ, Rodrigues L, Nascimento M, Marques T. Sequencebased and monoclonal antibody typing of Legionella pneumophila isolated from patients in Portugal during 1987-2008. Euro Surveill. 2009;14(28).

21. Kozak NA, Benson RF, Brown E, Alexander NT, Taylor TH, Shelton BG et al. Distribution of lag-1 Alleles and Sequence-Based Types among Legionella pneumophila Serogroup 1 Clinical and Environmental Isolates in the United States. J Clin Microbiol. 2009; 2525-2535.

22. Galli MG, Bianchi A, Raimondi A, Tesauro M, Consonni M. Use of three molecular methods for investigations of Legionnaires' disease in a hospital in Milan. J Hosp Infect. 2008; 69: 403-405.

23. Bianchi A, Tesauro M, Consonni M, Galli MG. Investigation on strains of Legionella pneumophila, isolated from a hospital of Milano, with three genotyping methods. Ann Ig. 2009; 21(5): 517-522.

24. Bianchi A, Pregliasco F, Consonni M, Tesauro M, Galli MG. New sequence types of Legionella pneumophila circulating in northern Italy and comparison with other regions of the world. J Hosp Infect. 2010; 76: 365-367.

25. European Working Group for Legionella Infections. The EWGLI SBT database for the typing of Legionella pneumophila (accessed dec 2014). Available from: http://www.hpa-bioinformatics.org.uk/legionella/ legionella_sbt/php/sbt_homepage.php

26. Chiarini A, Bonura C, Ferraro D, Barbaro R, Calà C, Distefano S, Casuccio N, Belfiore S, Giammanco A. Genotyping of Legionella pneumophila serogroup 1 strains isolated in Northen Sicily, Italy New Microbiologica. 2008; 31 (2): 217-228.

27. Ditommaso S, Giacomuzzi M, Arauco Rivera SR, Raso R, Ferrero P, Zotti CM. Virulence of Legionella pneumophila strains isolated from hospital water system and healthcare-associated Legionnaires' disease in Northen Italy between 2004 and 2009. BMC Infect Dis. 2014; 14: 483-490.

28. Rota MC, Caporali MG, Napoli C, Bella A, Giannitelli S, Scaturro M, Fontana S e Ricci ML. Rapporto annuale sulla legionellosi in Italia nel 2013. Not Ist Super Sanità 2014; 27 (10): 3-10.

29. Lin YE, Stout JE, Yu VL. Prevention of hospital-acquired legionellosis. Curr Opin Infect Dis. 2011; 24:3 50-356.

30. Sikora A, Wojtowicz Bobin M, Koziol-Montewka M, Magrys A, Gladysz I. Prevalence of Legionella pneumophila in water distribution systems in hospitals and public buildings oft he Lublin region of eastern Poland. Ann Agric Environ Med. 2015; 22(2): 195-201.

31. Allegheny County Health Department Approches to prevention and control of Legionella infection in Allegheny County Health Care Facilities. In 2nd edition. Pittsburgh PA: Allegheny County Health Department; 1997.

32. CDC Guidelines for preventing health care associated pneumonia MMWR Morb Mort Wkly Rep. 2003, 53: 1-36. 